\title{
Massive efflux of adenosine triphosphate into the extracellular space immediately after experimental traumatic brain injury
}

\author{
NOBUHIRO MORO ${ }^{1,2}$, SIMA S. GHAVIM ${ }^{1}$ and RICHARD L. SUTTON ${ }^{1}$ \\ ${ }^{1}$ Brain Injury Research Center, Department of Neurosurgery, David Geffen School of Medicine, \\ University of California, LA 90095-6901, USA; ${ }^{2}$ Department of Neurological Surgery, \\ Nihon University School of Medicine, Tokyo 173-8610, Japan
}

Received August 18, 2020; Accepted February 2, 2021

DOI: $10.3892 /$ etm.2021.10007

\begin{abstract}
The aim of the current study was to determine effects of mild traumatic brain injury (TBI), with or without blockade of purinergic ATP Y1 (P2Y1) receptors or store-operated calcium channels, on extracellular levels of ATP, glutamate, glucose and lactate. Concentrations of ATP, glutamate, glucose and lactate were measured in cerebral microdialysis samples obtained from the ipsilateral cortex and underlying hippocampus of rats with mild unilateral controlled cortical impact (CCI) or sham injury. Immediately after CCI, a large release of ATP was observed in the cortex (3.53-fold increase of pre-injury value) and hippocampus (2.97-fold increase of pre-injury value), with ATP returning to the baseline levels within 20 min post-injury and remaining stable for during the 3 -h sampling period. In agreement with the results of previous studies, there was a significant increase in glutamate 20 min after CCI, which was concomitant with a decrease in extracellular glucose (20 min) and an increase in lactate (40-60 min) in both brain regions after CCI. Addition of a selective P2Y1 receptor blocker (MRS2179 ammonium salt hydrate) to the microdialysis perfusate significantly lowered pre-injury ATP and glutamate levels, and eliminated the post-CCI peaks. Addition of a blocker of store-operated calcium channels [2-aminoethoxy diphenylborinate (2-APB)] to the microdialysis perfusate significantly lowered pre-injury ATP in the hippocampus, and attenuated the post-CCI peak in both the cortex and hippocampus. 2-APB
\end{abstract}

Correspondence to: Dr Nobuhiro Moro, Department of Neurological Surgery, Nihon University School of Medicine, 30-1 Oyaguchikamicho, Itabashiku, Tokyo 173-8610, Japan

E-mail: moro.nobuhiro@nihon-u.ac.jp

Abbreviations: ANCOVA, analysis of covariance; 2-APB, 2-aminoethoxy diphenylborinate; CCI, controlled cortical impact; MRS2179, MRS2179 ammonium salt hydrate; P2Y1, purinergic ATP Y1; TBI, traumatic brain injury

Key words: adenosine triphosphate, gliotransmitter, microdialysis, rat, traumatic brain injury treatment significantly increased baseline glutamate levels, but the values post-injury did not differ from those in the sham group. Pre-injury glucose levels, but not lactate levels, were increased by MRS2179 and decreased by 2-APB. However, none of these treatments substantially altered the CCI-induced reduction in glucose and increase in lactate in the cortex. In conclusion, the results of the present study demonstrated that a short although extensive release of ATP immediately after experimental TBI can be significantly attenuated by blockade of $\mathrm{P} 2 \mathrm{Y} 1$ receptors or store-operated calcium channels.

\section{Introduction}

Previous studies (1-17) indicated the importance and various roles of ATP signaling in the brain. Astrocytes, neurons and microglia are known to use ATP as a transmitter molecule by releasing it into the extracellular space to communicate with other cells $(1,2)$. ATP signaling has been reported to participate in synaptic strength (3-5) and memory formation by retaining long-term potentiation $(6,7)$, and can alter cerebral blood flow by increasing calcium levels in astrocytic endfeet, which increases adjacent brain vessel diameter (8-10). The most investigated function of ATP signaling is the activation of microglia to initiate inflammation (11-14). When microglia receive an ATP signal through their surface purinergic receptor, microglia change to amoeboid form, thus initiating the migration to the damaged location and phagocytosis of debris in the brain $(12,15,16)$. Moreover the well-known intercellular energy role of ATP in maintaining synaptic function, which is usually derived from glycolysis and lactate when high synaptic activity is ongoing (17), via ATP signaling in the extracellular space appears to be essential in both physiological and pathological brain.

Despite numerous in vitro studies showing various signaling functions of ATP in astrocytes, in vivo studies demonstrating ATP release in a disease model are limited. Melani et al (18) used cerebral microdialysis in a rat middle cerebral artery occlusion model and showed that the extracellular ATP value increased 1.3-fold compared with baseline levels during ischemia. Wang et al (19) detected an increase in ATP chemiluminescence by real-time imaging in a spinal cord injury model. Based on these previous in vivo and in vitro studies, combined with the well-known increase in extracellular glutamate after 
traumatic brain injury (TBI) (20), it was hypothesized that ATP should increase in the extracellular spaces acutely after controlled cortical impact (CCI)-induced mild injury. If ATP release occurs after TBI, this signal might be responsible for the inflammatory response after TBI that aggravates outcome, and could possibly be a treatment target. The present study used a cerebral microdialysis technique in a rat CCI model and measured the magnitude of ATP release concomitant with changes in extracellular glutamate. The effects of a blocker of the purinergic ATP Y1 (P2Y1) receptor and a blocker of the store-operated calcium channel that increases intracellular calcium levels were also investigated. Rapid glutamate release and energy substance (e.g, glucose, lactate) changes are well known to occur after TBI. The present study also measured glucose and lactate levels from the perfusate to determine their changes post-injury when the P2Y1 receptor or store-operated calcium channels were chemically suppressed.

\section{Materials and methods}

Ethics. All experimental procedures were approved by the UCLA Chancellor's Committee for Animal Research prior to starting the experiments. The approval protocol number was 2008-021. All experiments were conducted at UCLA. Efforts were made to minimize causing pain and distress to the animals.

Animal model. Thirty eight male Sprague-Dawley rats weighing between 313 and $413 \mathrm{~g}$ (mean, $355 \mathrm{~g}$; 62-72 day of age) were obtained from Charles River Laboratories, Inc., and were used in the study. Four rats were used to determine that our selected injury parameters did not result in cortical bleeding or tissue necrosis 1 week post-CCI and 8 rats were used in pilot work to determine the need for an inhibitor of ATP breakdown in the microdialysis perfusate. For animals used in the main experiment, 1 rat died shortly after induction of anesthesia with rapid rise in body temperature and 1 died from apparent neurogenic pulmonary complications shortly after CCI. Rats were pair housed and acclimated for at least 2 weeks under standard temperature and lighting conditions $\left(21-24^{\circ} \mathrm{C}\right.$, 30-70\% humidity, room lights on 6:00 a.m. - 6:00 p.m.), with food (Teklad; Envigo) and tap water available ad libitum. Prior to surgery, the animals were randomly assigned to one of four treatment groups: i) Sham injury group with standard perfusate (sham, $n=6$ ); ii) CCI group with standard perfusate (CCI; $n=6)$; iii) CCI group with in situ administration of $300 \mu \mathrm{M}$ MRS2179 ammonium salt hydrate (M3808; Sigma-Aldrich; Merck KGaA) (CCI-MRS2179; n=6); and iv) CCI group with in situ administration of $1 \mathrm{mM} 2$-aminoethoxy diphenylborinate (2-APB; D9754; Sigma-Aldrich; Merck KGaA) (CCI-2-APB; n=6). For both drug treated groups the compounds were administered by diffusion from the microdialysis probe membranes. Drug concentrations are determined from the previous study and considering the diffusion effect as explained below.

For surgery, the animal was placed in an anesthesia box and anesthesia was induced with 3-4\% isoflurane in oxygen (1.5 1/min flow rate) and the scalp was shaved. After the head was secured in a stereotaxic frame, anesthesia was maintained with 1.5-2.0\% isoflurane in oxygen delivered via nose cone on the stereotaxic bite-bar throughout surgery. Body temperature was kept at $37 \pm 0.5^{\circ} \mathrm{C}$ throughout surgery with a rectal probe and a thermostatically controlled heating pad (Harvard Apparatus). Ophthalmic ointment (Bausch \& Lomb) was applied to both eyes, local anesthesia was applied to the scalp with $0.5 \mathrm{ml} 1 \%$ lidocaine, and the scalp was sterilized by repeated (three times) cleaning with Betadine (Dynarex Corp.) followed by $70 \%$ ethanol. A midline skin incision was made, and the pericranium was spread bilaterally. Next, a 6-mm diameter circular craniotomy was made over the left parietal cortex in all animals, centered $3.0 \mathrm{~mm}$ posterior and $3.5 \mathrm{~mm}$ lateral from Bregma (Fig. 1). Under microscopic guidance (M651; Leica Microsystems $\mathrm{GmbH}$ ) the dura mater was incised at two sites with the tip of a 27 -gauge needle. Two microdialysis probes mounted on a single micromanipulator on the stereotaxic instrument (Kopf Instruments) were placed into the left hemisphere. One probe was placed into the cortex adjacent to the location of CCI. Another probe was placed into the hippocampus, which is underneath the impact site and known to exhibit several injury-induced changes. The final probe locations were selected with limitation of placement through a single craniotomy and use of a single micromanipulator. With minor deviations to avoid surface blood vessels, the target coordinates for the cortical probe were $1.5 \mathrm{~mm}$ posterior, $3.5 \mathrm{~mm}$ lateral from Bregma and $2.2 \mathrm{~mm}$ depth from cortical surface. The coordinates for the hippocampal probe were $3.5 \mathrm{~mm}$ posterior, $2.5 \mathrm{~mm}$ lateral from Bregma and $3.9 \mathrm{~mm}$ depth from cortical surface (Fig. 1). After 80 min of baseline sampling (collection of four 20-min fractions) both microdialysis probes were retracted by raising the micromanipulator until the probes were $\sim 10 \mathrm{~mm}$ above the cortical surface. Sham injury or CCI injury was then induced, similar to what is described in our prior studies (21-23) but with reduced tissue compression to induce a milder injury. A 4-mm diameter flat-tipped impactor, which was angled $20^{\circ}$ from vertical to be perpendicular to the dura mater at the injury site, was used to induce CCI centered at $3.0 \mathrm{~mm}$ posterior and $4.5 \mathrm{~mm}$ lateral relative to Bregma (20 psi, $2.37 \mathrm{~m} / \mathrm{sec}, 1.5 \mathrm{~mm}$ tissue compression for $250 \mathrm{msec}$ ). Pilot work in our laboratory showed that this injury level does not cause any gross evidence of tissue damage at 1 week post-CCI. Immediately after injury (within $\sim 10 \mathrm{sec}$ ), probes were returned to the same location by lowering the micromanipulator, and post-injury microdialysis sampling was performed for $3 \mathrm{~h}$ under isoflurane anesthesia. In total, the time of interruption of microdialysis sampling (probe retraction, CCI and probe re-insertion) was $<30 \mathrm{sec}$. The sham injury group underwent baseline sampling, as well as removal and replacement of probes, but without CCI application.

Microdialysis settings. Cerebral microdialysis was performed using CMA 12 Elite probes (8010431; CMA Microdialysis $\mathrm{AB}$ ), which have a 20,000 Da cut-off polyarylethersulfone membrane that is $1 \mathrm{~mm}$ in length with a $0.5-\mathrm{mm}$ membrane diameter. The perfusate used in all animals contained $147 \mathrm{mM}$

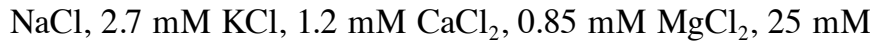
HEPES and $1 \mathrm{mM}$ adenosine 5'-( $\alpha, \beta$-methylene)diphosphate (M3763; Sigma-Aldrich; Merck KGaA) to prevent ATP breakdown (18). Pilot work $(n=8)$ showed that ATP levels in the extracellular fluid samples were not detectable if the ecto-5'-nucleotidase inhibitor [adenosine 5'-( $\alpha, \beta$-methylene $)$ diphosphate] 
was not contained in the perfusate. In one CCI group, $300 \mu \mathrm{M}$ MRS2179 was added to the perfusate to enable P2Y1 receptor blockade. For another CCI group, $1 \mathrm{mM} 2-\mathrm{APB}$ was mixed into the perfusate for blockade of store-operated calcium channels. Drug concentrations were selected to be 10 times higher than those used in previous in vitro experiments where MRS2179 or 2-APB were shown to suppress calcium responses (24-27), assuming $\sim 10 \%$ drug efflux from dialysate based on the probe membrane efficiency. Before use, the $\mathrm{pH}$ of each perfusate was adjusted to 7.4 and it was filtered through a sterile $0.2-\mu \mathrm{m}$ membrane. Prior to in vivo use, the efficiency of each probe was measured by collecting dialysates as the probe was immersed in a $10-\mu \mathrm{M}$ glutamate and $10-\mathrm{nM}$ ATP solution. During the experiments, each probe was perfused with perfusate at $3 \mu \mathrm{l} / \mathrm{min}$, and timing of sample collection was performed to account for fluid volumes contained in probe inlet and outlet tubes and the shaft dead space. All microdialysis samples were collected in 20-min fractions, with four samples collected in an 80-min baseline sampling period and nine samples collected in $3 \mathrm{~h}$ of post-injury sampling. All microdialysis samples were stored at $-80^{\circ} \mathrm{C}$ until analysis. Both ATP and glutamate data are presented as values corrected by the percentage of recovery of each probe. The percentage of recovery of glutamate was substituted for correcting both glucose and lactate values. At the end of surgery, a $0.5 \mathrm{ml}$ venous blood sample was collected from the subcutaneous vein of the neck to enable determination of plasma glucose and lactate concentrations. The isoflurane-anesthetized animals were sacrificed by decapitation, and their brains were post-fixed in $4 \%$ paraformaldehyde and then sectioned in a cryostat to enable histological staining.

Sample analyses. The concentration of ATP in each cerebral microdialysis fraction was measured by a bioluminescent luciferin-luciferase assay using a commercial kit (FLAAM and FLAAB; Sigma-Aldrich; Merck KGaA) according to the manufacturer's protocol. The signal was read with a scintillation counter (LS6500; Beckman Coulter, Inc.). Glutamate, glucose and lactate concentrations were measured using a CMA 600 analyzer (8001600; CMA Microdialysis AB). Plasma glucose and lactate levels were analyzed with YSI 2700 Select Biochemistry Analyzer (Yellow Springs Instrument; Thermo Fisher Scientific, Inc.).

Statistical analysis. For statistical analysis, SPSS version 21 software (IBM Corp.) was used. All data are presented as the group mean \pm standard deviation. Student's t-test was used to compare data for body temperature and blood glucose or lactate levels between the sham and CCI groups. For statistical analysis of the pre-injury microdialysis values, one-way ANOVA was applied to the values of the final samples in the baseline period (-20 min samples). When significant differences in the baseline data were obtained across groups (including ATP, glutamate and glucose values), the post-injury data were analyzed using repeated measures analysis of covariance (ANCOVA) with each pre-injury value of individual subjects serving as covariates. For data without baseline difference (such as lactate values), repeated measures ANOVA was used to analyze the post-injury data. When the post-injury analyses revealed significant differences across the groups, post hoc Tukey's test was conducted for multiple comparisons. $\mathrm{P}<0.05$ was considered to indicate a statistically significant difference.

\section{Results}

Location of probes and physiological parameters. As aforementioned, to avoid cortical surface vessels, microdialysis probes could not be inserted into the planned position for certain animals. The final cortical probe position on average was $1.62 \pm 0.29 \mathrm{~mm}$ posterior and $3.53 \pm 0.33 \mathrm{~mm}$ lateral from Bregma, and the final hippocampal probe position was $3.62 \pm 0.29 \mathrm{~mm}$ posterior and $2.53 \pm 0.33 \mathrm{~mm}$ lateral from Bregma. These probe locations were very close to the initial targets, and did not alter placement in the intended cortical or hippocampal tissues.

The mean lowest and highest body temperature values during surgery in the sham group were respectively $36.07 \pm 0.29^{\circ} \mathrm{C}$ and $36.87 \pm 0.64^{\circ} \mathrm{C}$, and $36.07 \pm 0.27^{\circ} \mathrm{C}$ and $36.97 \pm 0.61^{\circ} \mathrm{C}$ in the $\mathrm{CCI}$ group. The mean blood glucose levels at $3 \mathrm{~h}$ post-injury were $10.29 \pm 1.05 \mathrm{mM}$ in the sham group and $10.53 \pm 0.86 \mathrm{mM}$ in the CCI group. The mean blood lactate levels at the same time point were $1.27 \pm 0.31 \mathrm{mM}$ in the sham group and $1.15 \pm 0.19 \mathrm{mM}$ in the CCI group. These parameters were not significantly different between groups.

ATP changes in the extracellular space. The ATP changes in the extracellular spaces are represented in Fig. 2A (cortex) and Fig. 2B (hippocampus).

The final (-20 min) pre-injury dialysate ATP values in the cortex were $8.71 \pm 0.97 \mathrm{nM}$ in the sham group, $8.50 \pm 0.20 \mathrm{nM}$ in the CCI group and 7.59 $\pm 1.00 \mathrm{nM}$ in the CCI-2-APB group. The CCI-MRS2179 group had a significantly lower value $(2.73 \pm 0.17 \mathrm{nM})$ compared with that of the other three groups $(\mathrm{P}<0.001)$, indicating significant effects of $\mathrm{P} 2 \mathrm{Y} 1$ receptor blockade on pre-injury ATP release. In the first $20 \mathrm{~min}$ following injury, the ATP value of the CCI group increased to $30.01 \pm 6.95 \mathrm{nM}$ which was significantly higher $(\mathrm{P}<0.001)$ than that of the sham group $(11.53 \pm 2.07 \mathrm{nM})$. The ATP concentration of the CCI group increased by $353 \%$ from the pre-injury value, while that of the sham group was $132 \%$ of pre-injury value. The ATP value of the CCI-MRS2179 group was not significantly altered by injury, with a concentration of $2.98 \pm 0.16 \mathrm{nM}$ at $20 \mathrm{~min}$ post-CCI $(109 \%$ of pre-injury value). The ATP value of the CCI-2-APB group increased to $19.41 \pm 3.84 \mathrm{nM}(256 \%$ of pre-injury value) in the first $20 \mathrm{~min}$ after injury, but this increase was not significantly different from that of the sham group. Both the CCI-MRS2179 and CCI-2-APB groups exhibited significantly $(\mathrm{P}=0.001)$ lower post-injury peak ATP values compared with those of the CCI group. The ATP values of the four groups returned to their baseline levels within the second post-injury fraction, with no further significant group differences.

The ATP changes in the hippocampus exhibited similar trends to those of the cortex, with the greatest changes occurring in the first post-injury microdialysis fraction (Fig. 2B). In the sham group, the ATP value changed from $8.47 \pm 0.22$ to $11.43 \pm 1.50 \mathrm{nM}$ ( $135 \%$ of pre-injury value). In the CCI group, the pre-injury value of $8.59 \pm 0.40 \mathrm{nM}$ increased to $25.49 \pm 4.92 \mathrm{nM}$ ( $297 \%$ of pre-injury value), and this peak value was significantly higher $(\mathrm{P}<0.001)$ than that of the sham group. 


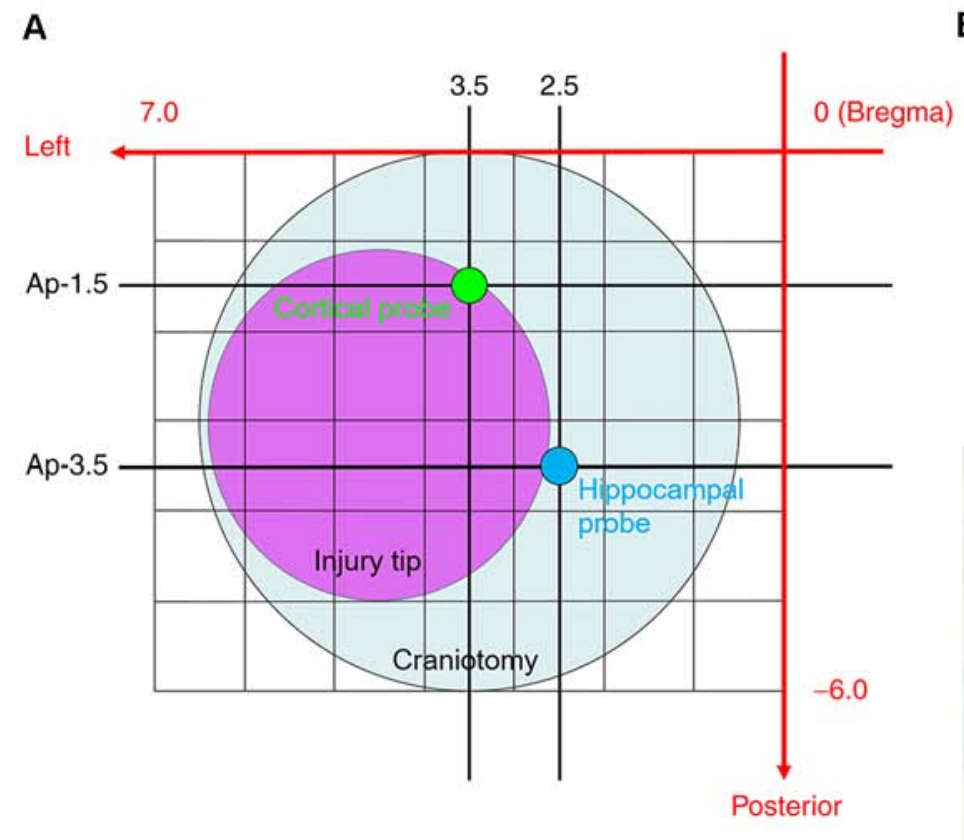

B

(1) $\rightarrow$

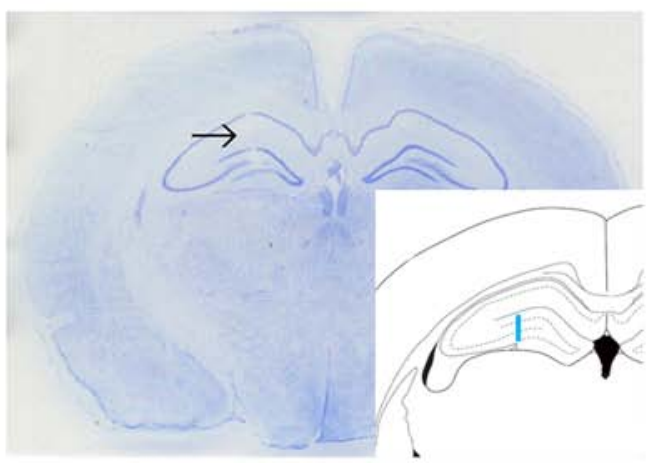

Figure 1. (A) Schematic diagram depicting anterior-posterior locations of the craniotomy (light blue), the injury tip (purple) and target surface sites for the cortex and hippocampal microdialysis probes relative to Bregma. (B) Two thionin-stained histology images (magnification, $\mathrm{x} 5$ ) indicate the actual probe locations (arrows) in the cortex (top) and hippocampus (bottom). Cerebral contused tissue was not observed in this mild controlled cortical impact injury model. The brain atlas images depict the locations of the working membrane of the cortical and hippocampal microdialysis probes.

The -20 min pre-injury ATP value of the CCI-MRS2179 group was significantly reduced $(\mathrm{P}<0.001)$ compared with that of the other three groups. In this group, the ATP value changed from $2.88 \pm 0.07 \mathrm{nM}$ pre-injury to $3.05 \pm 0.21 \mathrm{nM}$ (106\% of pre-injury value) in $20 \mathrm{~min}$ after CCI, with a significantly lower peak value compared with that of the sham $(\mathrm{P}<0.001)$ and CCI $(\mathrm{P}<0.001)$ groups. The pre-injury ATP value in hippocampus of the CCI-2-APB group was significantly lower $(\mathrm{P}<0.001)$ than that of the sham and CCI groups. The ATP values of the CCI-2-APB group changed from $7.40 \pm 0.41 \mathrm{nM}$ pre-injury to $17.25 \pm 4.26 \mathrm{nM}(233 \%$ of pre-injury value $)$, and the $20 \mathrm{~min}$ post-CCI peak value was significantly lower than that of the CCI group $(\mathrm{P}<0.001)$. From the second post-injury fraction onward, the ATP value of all groups returned to the baseline level without any significant difference between the groups.

Glutamate changes in the extracellular space. The glutamate changes in the extracellular spaces are represented in Fig. 3A (cortex) and Fig. 3B (hippocampus).

In the cortex, the -20 min pre-injury glutamate levels of the sham and CCI groups were 13.29 3.18 and $12.49 \pm 1.38 \mu \mathrm{M}$, respectively. The pre-injury glutamate value of the CCI-MRS2179 group $(9.13 \pm 1.82 \mu \mathrm{M})$ was significantly reduced $(\mathrm{P}=0.030)$, while that of the CCI-2-APB group $(23.70 \pm 2.12 \mu \mathrm{M})$ was significantly increased $(\mathrm{P}<0.001)$, compared with that of the sham group, demonstrating the pre-injury effects of P2Y1 receptor or store operated calcium channel blockade. In the first fraction after injury, the cortical glutamate concentration of the CCI group reached $51.63 \pm 17.18 \mu \mathrm{M}(413 \%$ of pre-injury value), which was significantly higher $(\mathrm{P}<0.001)$ than the concentration observed in the sham group (93\% of pre-injury value; $12.38 \pm 3.01 \mu \mathrm{M})$. The glutamate values for the CCI-MRS2179 and CCI-2-APB groups increased to $29.71 \pm 4.46 \mu \mathrm{M}(325 \%$ of pre-injury value) and $36.39 \pm 8.23 \mu \mathrm{M}$ (154\% of pre-injury value), respectively, in the first $20 \mathrm{~min}$ post-injury, but these values were not significantly different from those of the sham group. The post-injury peak glutamate values of the CCI-MRS2179 group were significantly lower $(\mathrm{P}=0.037)$ than those of the CCI group. In the second fraction after injury, the cortical glutamate values of the four groups returned to the baseline level, with no further significant group differences.

In the hippocampus (Fig. 3B), the -20 min pre-injury glutamate levels were $12.78 \pm 1.37$ and $12.67 \pm 0.61 \mu \mathrm{M}$ in the sham and CCI groups, respectively. In the cortex, the pre-injury value of the CCI-MRS2179 group $(9.22 \pm 1.61 \mu \mathrm{M})$ was significantly lower $(\mathrm{P}=0.027)$, and the pre-injury value of the CCI-2-APB group $(20.27 \pm 3.31 \mu \mathrm{M})$ was significantly higher $(\mathrm{P}<0.001)$ in the hippocampus than the pre-injury glutamate levels in the sham group. In the first fraction following injury, the peak value of the CCI group increased significantly (260\% of pre-injury value; $32.98 \pm 12.32 \mu \mathrm{M} ; \mathrm{P}=0.040)$ compared with that of the sham group ( $91 \%$ of pre-injury value; $11.61 \pm 1.53 \mu \mathrm{M})$. The post-injury glutamate peak for the CCI-MRS2179 group was $21.30 \pm 4.74 \mu \mathrm{M}(231 \%$ of pre-injury value), and that of the CCI-2-APB group was $47.64 \pm 19.94 \mu \mathrm{M}$ (235\% of pre-injury value), with neither of these increases being significant compared with the values exhibited by the sham group. In the second fraction post-injury and thereafter, the values for glutamate in the hippocampus of the four groups returned to the baseline level without any significant group differences.

The post-injury glutamate peaks of the MRS2179 and 2 -APB groups increased to $154-325 \%$ of pre-injury value in the cortex and hippocampus, but ANCOVA showed that these 

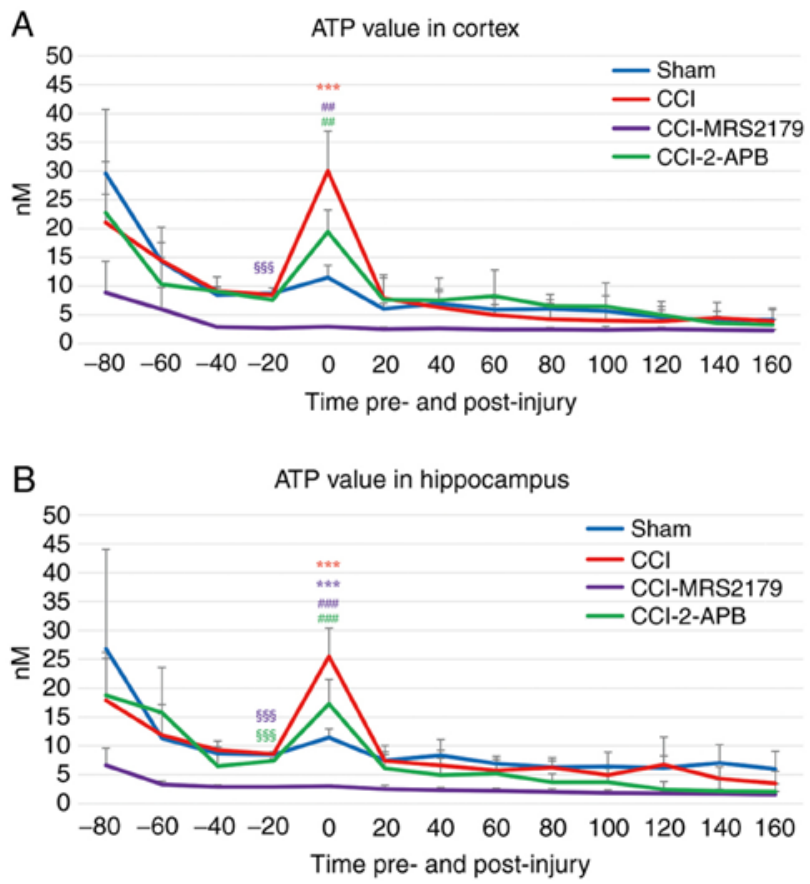

Figure 2. ATP levels in the extracellular space in the (A) cortex and (B) hippocampus. The times shown for each 20-min dialysate represent the start of each sample collection relative to the time of injury. Low ATP baseline (-20 min pre-injury) levels were noted in the CCI-MRS2179 (cortex and hippocampus) and CCI-2-APB (hippocampus) groups compared with those of the sham injury controls. Large release of ATP was observed immediately after mild CCI injury in both the cortex and hippocampus. This increase was significantly attenuated by either MRS2179 or 2-APB treatment. The ATP values returned to the -20 min pre-injury levels at 20-40 min after injury. The color of each symbol denoting significant group differences correspond to the colors of the lines for each group shown in the legend. ${ }^{\$ \$} \mathrm{P}<0.001$ vs. the sham group at -20 min pre-injury. ${ }^{* * *} \mathrm{P}<0.001$ vs. the sham group post-injury. ${ }^{\# \#} \mathrm{P}<0.01$ and ${ }^{\# \# \#} \mathrm{P}<0.001$ vs. the $\mathrm{CCI}$ group post-injury. 2-APB 2-aminoethoxy diphenylborinate; CCI, controlled cortical impact; MRS2179, MRS2179 ammonium salt hydrate.

changes were not significantly different compared with the values exhibited by the sham group $(\mathrm{P}=0.148$ in cortex and $\mathrm{P}=0.990$ in hippocampus). The significance of these post-CCI peak values was eliminated due to the significant $-20 \mathrm{~min}$ pre-injury levels being set as a covariate in these groups.

Glucose changes in the extracellular space. The glucose concentrations in the extracellular space are represented in Fig. 4A (cortex) and Fig. 4B (hippocampus).

The -20 min pre-injury glucose level in the cortex of the sham group was $1.02 \pm 0.07 \mu \mathrm{M}$, and in the CCI group it was $1.04 \pm 0.05 \mu \mathrm{M}$. The CCI-MRS2179 group had significantly higher $(\mathrm{P}=0.004)$ pre-injury glucose value $(1.23 \pm 0.13 \mu \mathrm{M})$ and the CCI-2-APB group had a significantly lower $(\mathrm{P}<0.001)$ pre-injury glucose value $(0.77 \pm 0.08 \mu \mathrm{M})$ compared with those of the sham controls. After injury, in the first fraction, the glucoses value decreased to $0.70 \pm 0.11$ (67\% of pre-injury value), $0.57 \pm 0.19$ ( $47 \%$ of pre-injury value) and $0.42 \pm 0.06 \mu \mathrm{M}$ (54\% of pre-injury value) in the CCI, CCI-MRS2179 and CCI-2-APB groups, respectively. These post-CCI values were significantly reduced $(\mathrm{P}<0.001)$ compared with those of the sham group (106\% of pre-injury value; $1.09 \pm 0.06 \mu \mathrm{M})$. The cortical glucose values of the CCI group returned to near baseline from the second post-injury fraction onward, without any significant differences versus the sham group. The cortical glucose values of the CCI-MRS2179 group started to recover after the second fraction post-injury and became significantly higher compared with those of the sham group at 100-120 min $(\mathrm{P}=0.016)$ and at $160-180 \mathrm{~min}$ after injury $(\mathrm{P}=0.041)$. The glucose level in cortical dialysates of the CCI-2-APB group started to recover from the second fraction post-injury but remained significantly lower than the levels in the sham controls at 20-40 $\min (\mathrm{P}=0.004)$ and $40-60 \mathrm{~min}(\mathrm{P}=0.001)$ after CCI.

The hippocampal glucose levels of the four groups showed similar trends to those observed in cortex (Fig. 4B). The -20 min pre-injury glucose concentrations in the sham and CCI groups were $0.97 \pm 0.07$ and $1.00 \pm 0.12 \mu \mathrm{M}$, respectively. The CCI-MRS2179 group had significantly higher $(\mathrm{P}<0.001)$ glucose values $(1.23 \pm 0.07 \mu \mathrm{M})$, and the CCI-2-APB group had significantly lower $(\mathrm{P}=0.010)$ glucose values $(0.79 \pm 0.07 \mu \mathrm{M})$ compared with those of the sham group. A post-injury decrease was observed in extracellular glucose in the hippocampus of the CCI group (75\% of pre-injury value; $0.75 \pm 0.07 \mu \mathrm{M})$, which was significant $(\mathrm{P}=0.001)$, compared with that of the sham group $(106 \%$ of pre-injury value; $1.02 \pm 0.16 \mu \mathrm{M})$. In the CCI-MRS2179 group, glucose levels decreased to $1.03 \pm 0.10 \mu \mathrm{M}$ (84\% of pre-injury value) in the first post-injury fraction, which did not differ from those of the sham group. The first $20 \mathrm{~min}$ post-injury glucose levels of the CCI-2-APB group were $0.62 \pm 0.05 \mu \mathrm{M}$ (79\% of pre-injury value), which were significantly reduced $(\mathrm{P}=0.002)$ compared with those in the sham group. In the second fraction post-injury, the glucose levels in hippocampal dialysates returned to baseline level ranges, with no significant difference between the four groups thereafter.

Lactate changes in the extracellular space. The lactate data from the cerebral microdialysis samples are represented in Fig. 5A (cortex) and Fig. 5B (hippocampus).

The -20 min pre-injury lactate levels in the cortex of the sham, CCI, CCI-MRS2179 and CCI-2-APB groups were $1.20 \pm 0.04,1.21 \pm 0.07,1.02 \pm 0.42$ and $1.05 \pm 0.07 \mu \mathrm{M}$, respectively. There was no significant difference between the four groups for pre-injury lactate levels. In the CCI group, the post-injury cortical lactate value reached $5.44 \pm 1.73 \mu \mathrm{M}$ (449\% of pre-injury value) in the first fraction and $3.67 \pm 2.06 \mu \mathrm{M}$ in the second fraction, with these values being significantly different $(\mathrm{P}<0.001$ and $\mathrm{P}=0.037$, respectively) compared with the sham group values of $1.54 \pm 0.19 \mu \mathrm{M}$ (129\% of pre-injury value) and $1.41 \pm 0.15 \mu \mathrm{M}$. In the CCI-MRS2179 group, cortical lactate in the first post-injury fraction was $5.98 \pm 1.13 \mu \mathrm{M}$ (588\% of pre-injury value), and it was $3.99 \pm 1.29 \mu \mathrm{M}$ in the second fraction. Both values were significantly elevated $(\mathrm{P}<0.001$ and $\mathrm{P}=0.021$, respectively) compared with the findings in the sham group. The lactate value of the CCI-2-APB group increased significantly $(\mathrm{P}=0.001)$ above sham levels to $4.44 \pm 0.78 \mu \mathrm{M}$ ( $442 \%$ of pre-injury value) in the first fraction, but it was not significantly different from that of the sham group in the second fraction post-injury.

In the hippocampus, the -20 min pre-injury lactate values of the sham, CCI, CCI-MRS2179 and CCI-2-APB groups were $1.25 \pm 0.04,1.26 \pm 0.15,1.25 \pm 0.04$ and $1.22 \pm 0.11 \mu \mathrm{M}$, 

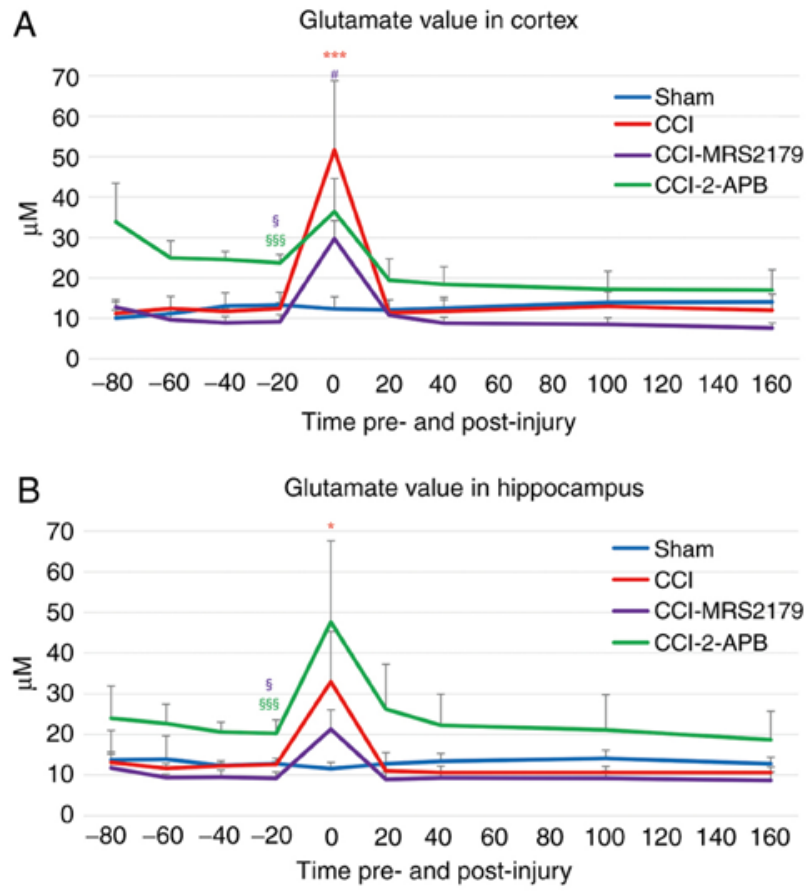

Figure 3. Glutamate levels in the extracellular space in the (A) cortex and (B) hippocampus. The times shown for each 20-min dialysate represents the start of each sample collection relative to the time of injury. Low baseline (-20 min pre-injury) glutamate levels in the CCI-MRS2179 group and high baseline levels in the CCI-2-APB group compared with those of the sham controls were observed in both brain regions. A large release of glutamate was observed in the CCI group immediately after injury in both the cortex and hippocampus. This glutamate release was attenuated in the CCI-MRS2179 and CCI-2-APB groups. The color of each symbol denoting significant group differences correspond to the colors of the lines for each group shown in the legend. ${ }^{\S} \mathrm{P}<0.05$ and ${ }^{\$ \$} \mathrm{P}<0.001$ vs. the sham group at -20 min pre-injury. ${ }^{*} \mathrm{P}<0.05$ and ${ }^{* * *} \mathrm{P}<0.001$ vs. the sham group post-injury. ${ }^{\#} \mathrm{P}<0.05$ vs. the $\mathrm{CCI}$ group post-injury. 2-APB, 2-aminoethoxy diphenylborinate; $\mathrm{CCI}$, controlled cortical impact; MRS2179, MRS2179 ammonium salt hydrate.

respectively (Fig. 5B). There was no significant difference between the four groups for pre-injury lactate. In the CCI group, the lactate concentrations in the hippocampus increased to $3.14 \pm 0.63$ ( $249 \%$ of pre-injury value), $2.16 \pm 0.60$ and $1.84 \pm 0.54 \mu \mathrm{M}$ in the first, second and third post-injury fractions, respectively, with these levels being significantly increased $(\mathrm{P}<0.001, \mathrm{P}=0.006$ and $\mathrm{P}=0.028$, respectively) compared with those of the sham group (114\% of pre-injury value; $1.42 \pm 0.17,1.24 \pm 0.22$ and $1.26 \pm 0.21 \mu \mathrm{M}$, respectively). In the CCI-MRS2179 group, the lactate values (212\% of pre-injury value; $2.65 \pm 0.48$ and $2.03 \pm 0.47 \mu \mathrm{M}$ ) were significantly higher $(\mathrm{P}<0.001$ and $\mathrm{P}=0.019)$ than those of the sham group for the first two post-injury fractions. In the CCI-2-APB group, the post-injury lactate levels in the hippocampus increased to $3.17 \pm 0.19 \mu \mathrm{M}$ (259\% of pre-injury value) in the first fraction, $2.39 \pm 0.30 \mu \mathrm{M}$ in the second fraction and $1.99 \pm 0.17 \mu \mathrm{M}$ in the third fraction. These values were all significantly higher than the lactate levels in the sham group $(\mathrm{P}<0.001, \mathrm{P}=0.001$ and $\mathrm{P}=0.005$, respectively).

\section{Discussion}

A major finding of the present study is that a large quantity of ATP was released into the extracellular spaces in the cortex
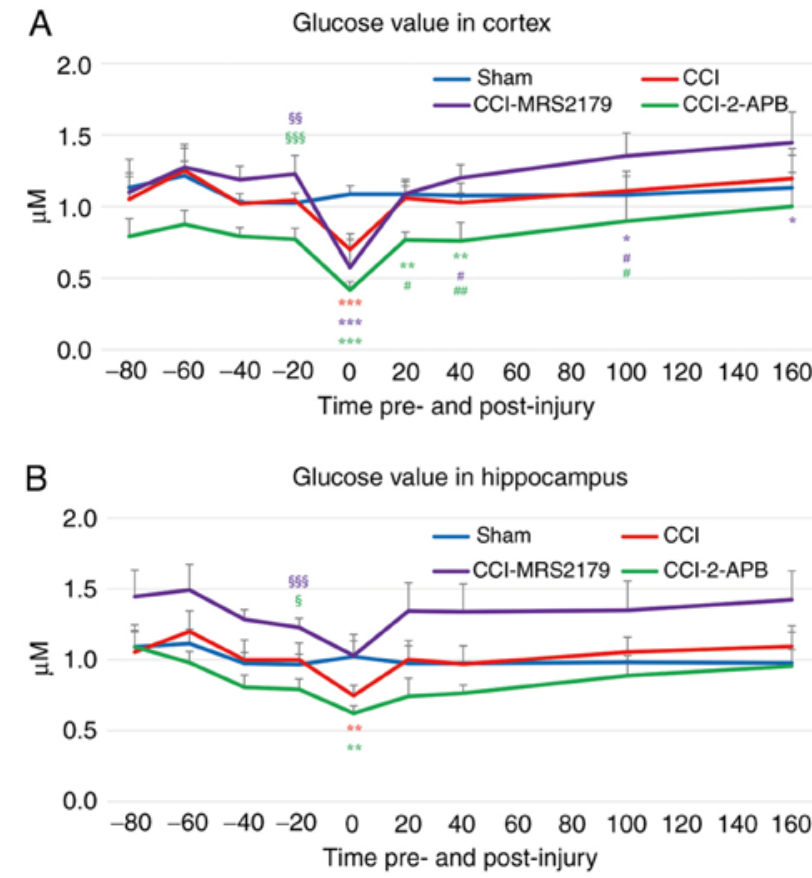

Figure 4. Glucose levels in the extracellular space in the (A) cortex and (B) hippocampus. The times shown for each 20-min dialysate represent the start of each sample collection relative to the time of injury. Administration of MRS2179 increased, while administration of 2-APB decreased, the baseline (-20 min pre-injury) glucose levels in both brain regions compared with those of the sham group. The significant injury-induced decrease in glucose vs. the sham controls did not differ between the three CCI groups in the first post-injury dialysate sample from the cortex, although this decrease in the hippocampus did not reach significance in the CCI-MRS2179 group. Additional long-term reductions in extracellular glucose with 2-APB treatment and increases with MRS2179 treatment were also observed in the cortex. The color of each symbol denoting significant group differences correspond to the colors of the lines for each group shown in the legend. ${ }^{\S} \mathrm{P}<0.05,{ }^{\S} \mathrm{P}<0.01$ and ${ }^{\S \S} \mathrm{P}<0.001$ vs. the sham group at -20 min pre-injury. ${ }^{*} \mathrm{P}<0.05,{ }^{* *} \mathrm{P}<0.01$ and ${ }^{* * *} \mathrm{P}<0.001$ vs. the sham group post-injury. ${ }^{*} \mathrm{P}<0.05$ and ${ }^{\# \#} \mathrm{P}<0.01$ vs. the CCI group post-injury. 2-APB, 2-aminoethoxy diphenylborinate; CCI, Controlled cortical impact; MRS2179, MRS2179 ammonium salt hydrate.

and hippocampus immediately after mild experimental TBI. In addition, it was demonstrated that this ATP release was restricted by in situ pre-injury administration of the P2Y1 receptor blocker MRS2179 or the store-operated calcium channel blocker 2-APB. Treatment with 2-APB increased the pre-injury extracellular glutamate level, but both MRS2179 and 2-APB reduced the extent of glutamate release induced by trauma. The pre-injury extracellular glucose level was increased by MRS2179 and decreased by 2-APB, but the post-CCI decrease in glucose levels was not altered by either P2Y1 or store-operated calcium channel blockade. Anaerobic metabolism, which is reflected by an increase in extracellular lactate after CCI, was not substantially affected by either MRS2179 or 2-APB treatment.

Although it is now recognized that a variety of brain cells release ATP (28-30), it seems likely that part of the increase in ATP observed in the present study derives from astrocytes. It is known that astrocytes increase their calcium levels and become activated astrocytes in a wide range including areas that are not directly damaged in response to environmental changes. In this process, gliotransmitters ATP, D-serine and 

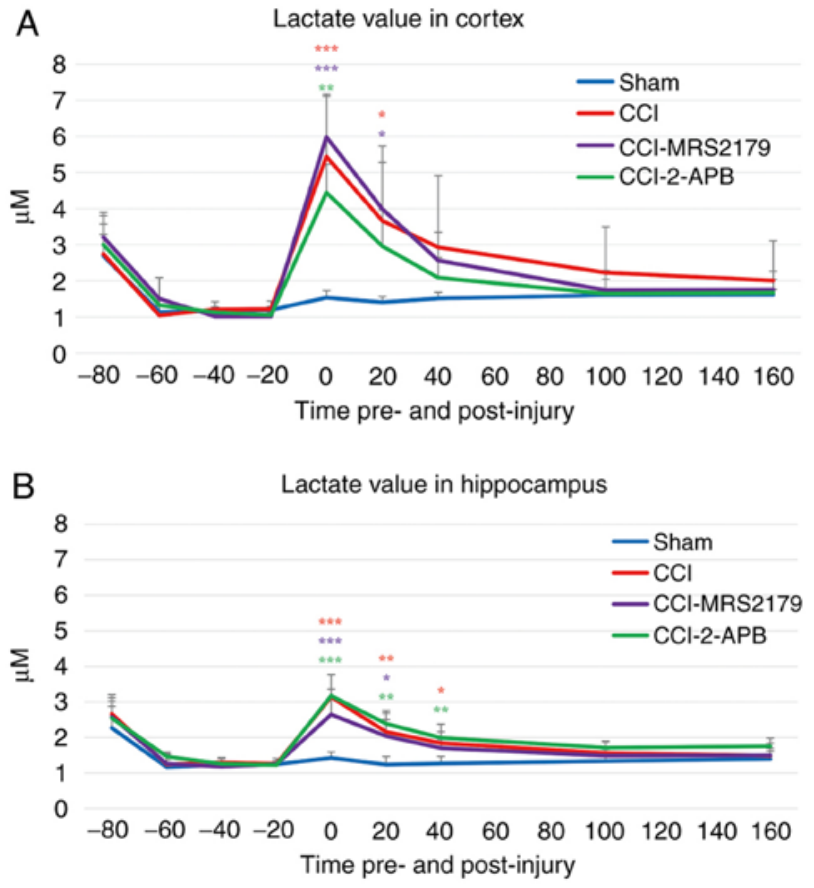

Figure 5. Lactate levels in the extracellular space in the (A) cortex and (B) hippocampus. The times shown for each 20 -min dialysate represent the start of each sample collection relative to the time of injury. Baseline (-20 min pre-injury) lactate levels did not differ between the four groups in either brain region. CCI injury produced a significant increase in lactate in the cortex and hippocampus compared with that of sham injury, although the injury-induced lactate increases did not differ between the three CCI groups. The color of each symbol denoting significant group differences correspond to the colors of the lines for each group shown in the legend. ${ }^{*} \mathrm{P}<0.05,{ }^{* *} \mathrm{P}<0.0$ and ${ }^{* * * *} \mathrm{P}<0.001$ vs. the sham group post-injury. 2-APB, 2 -aminoethoxy diphenylborinate; CCI, controlled cortical impact; MRS2179, MRS2179 ammonium salt hydrate.

glutamate are used as a paracrine signal to communicate with surrounding astrocytes, synapses and microglia. The $\mathrm{P} 2$ receptor that receives ATP signals is known to exist in the majority of brain cells. The major $\mathrm{P} 2$ receptor subtype to receive ATP signals during gliotransmission is known to be the P2Y1 receptor $(24,31)$. Molnar et al (32) reported that the P2Y1 receptor blocker MRS2179 successfully restricted gliotransmission in a rat brain slice model. In other studies, administration of MRS2179 restricted calcium increase and ATP release of astrocytes, while stimulation of $\mathrm{P} 2 \mathrm{Y} 1$ receptors potentiated ATP release $(33,34)$. To increase the intracellular calcium level, astrocytes release calcium from the endoplasmic reticulum or import calcium from the extracellular space through calcium channels on the cell membrane. In addition to the voltage dependent calcium channel, store-operated calcium channels that are activated by calcium depletion in the endoplasmic reticulum play an important role in ATP release (35). The blocker 2-APB has been shown to decrease calcium entry through the store-operated calcium channel, which results in the attenuation of calcium signaling (36).

Astrocytes are activated by several stimuli such as glutamate, ischemia and mechanical stress. Koizumi et al (37) applied mechanical stress to cultured astrocytes and demonstrated an increase in ATP bioluminescence signaling. Those results showed that mechanically stimulated astrocytes create a calcium wave that spreads concentrically at $21 \pm 1.7 \mu \mathrm{m} / \mathrm{sec}$.
It is important to clarify the role of astrocytes and their effects after TBI, since astrocytes play a complex role in several functions of the brain such as inflammation, blood flow regulation, metabolism, plasticity, epilepsy and mental disorders $(38,39)$. Of note, the source of ATP detected in the present study may derive from cells other than astrocytes, such as microglia, oligodendrocytes, Muller cells, Bergmann glia and neurons, which have all been reported to release ATP (28-30). In addition, P2Y1 receptors and store-operated calcium channels are not astrocyte specific.

The present results revealed that a large quantity of ATP was released into the extracellular space immediately after mild experimental TBI, which is consistent with previous studies on increased ATP signaling in other in vivo injury models $(18,19)$. However, the extracellular levels reported in the present study were markedly low compared with the reported ATP values within the cell (40). After injury, astrocytes probably release gliotransmitters to increase the activity of neighboring astrocytes and/or to initiate the microglial response to maintain electrolyte levels in the extracellular space and to remove debris from the brain $(12,15)$. Besides this beneficial effect of microglia, in TBI, it is known that the insult caused by trauma will cause excessive inflammation that can lead to a harmful secondary injury (41).

An unexpected finding of the present study is that MRS2179 reduced extracellular ATP within the baseline sampling period to an extremely low value, although this result is consistent with a previous study reporting that administration of MRS2179 decreased the frequency of astroglial calcium oscillation in a rat epilepsy model (42). The low levels of ATP in the CCI-MRS2179 group both pre- and post-injury suggest that P2Y1 receptor blockade may induce certain adverse effects if used long term, and future studies should examine short-term, post-injury treatment. Preventing the activation of astrocytes for therapeutic purpose needs to be carefully evaluated, since astrocytes have both beneficial and harmful aspects in TBI.

The present study also showed that the store-operated calcium channel was required to release ATP after relatively mild CCI injury. The store-operated calcium channel, together with the endoplasmic reticulum, is known to be essential for astrocytes to control the intracellular calcium level (43). The pre-injury ATP value of the CCI-2-APB group was significantly higher than that of the CCI-MRS2179 group, and it was similar to that of the sham and CCI groups. The 2-APB compound is known to block the activation of astrocytes in lipopolysaccharide-stimulated cultured astrocytes (44). The present results suggest that, in physiological conditions, P2Y1 signaling is required, but not the store-operated calcium channel, to maintain homeostasis for glutamate and electrolyte preservation. However, when greater signal transduction is required in response to an insult such as trauma, not only P2Y1 signaling but also the store-operated calcium channel are essential to release sufficient ATP signals into the extracellular space.

One limitation of the present study is the use of microdialysis for the study of ATP levels, since the mechanical insertion of the $0.5-\mathrm{mm}$ diameter probe into the brain parenchyma constitutes a stab wound injury. Astrocytes are known to release ATP and create a calcium wave even after mild mechanical stimuli to a single cell (45). In the current study the 
ATP values of the first fraction (-80 $\mathrm{min}$ ) of baseline sampling are considered to represent the effects of this stab wound. Notably, the ATP values were higher in all groups at that time compared with those at the final baseline (-20 min), and P2Y1 receptor blockade reduced ATP levels within the first $20 \mathrm{~min}$ of sampling period. The smaller post-injury ATP peak in the sham group likely represents ATP release due to further mechanical stimulation caused by retraction and re-insertion of probes. As this same stimulus was provided along with mild TBI to the CCI, CCI-MRS2179 and CCI-2-APB groups, post-injury ATP values must be interpreted with consideration of this 'probe injury' effect, with a portion of the total ATP peak observed post-injury being attributed to stimulation from probe re-insertion and a portion attributed to the mild TBI. The observed increased in ATP after CCI does not appear to simply reflect ATP leakage from mechanically injured cells, since the microdialysis probes were inserted into the cortex at the anterior edge of the contusion site as well as in the more remote, underlying hippocampus, and in both sampling sites the post-CCI ATP release was restricted by the P2Y1 receptor blocker and the store-operated calcium channel blocker. In the present study, a mild injury level that does not cause visible or histological contusion was used to minimize any ATP leakage from mechanically disrupted cells. The use of this mild TBI may explain why the peak in post-injury ATP levels was similar to the peak observed after the initial probe insertions for baseline sampling. Future studies should determine if the post-injury ATP peak is higher or more enduring to last longer if a moderate-to-severe TBI is administered at levels sufficient to cause cerebral contusion.

Consistent with previous studies on TBI $(20,46)$, extracellular glutamate was transiently increased in the current study. The results of the cortical microdialysis probe showed that inhibition of the P2Y1 receptor decreased the extracellular glutamate release after injury. Astrocytes are known to control the neuronal glutamate release in physiological brain $(47,48)$, and astrocytes themselves release glutamate as a transmitter into the extracellular space (49). MRS2179 probably inactivated astrocytes and neurons, which restricted glutamate release, thus resulting in lowered glutamate concentrations in the extracellular space. Glutamate toxicity is a well-known aggravating factor in cerebral infarct or TBI (50). Thus, reduced cortical glutamate with MRS2179 treatment suggests that modification of gliotransmission after injury may have a therapeutic potential to decrease secondary injury. It is also noteworthy that the pre-injury glutamate levels increased in the 2-APB-treated group. The increase in extracellular glutamate in the 2-APB-treated group may reflect that store-operated calcium channels are essential to re-pump the glutamate from the extracellular space, which may be associated with the function of metabotropic glutamate receptors (51).

The current results also confirm previous studies on early decrease in extracellular glucose with concomitant increase in extracellular lactate after brain trauma, which is likely to be due to increased glutamatergic activity with anaerobic glycolysis $(52,53)$. The baseline glucose and lactate levels in the present study were consistent with previously reported values, but were slightly lower compared with those of a freely moving rat microdialysis model $(54,55)$. The extracellular glucose levels were increased by MRS2179 treatment, although the relative reduction in glucose post-CCI was not altered by this P2Y1 receptor blockade. The lactate levels were not altered by MRS2179 treatment. The higher glucose concentrations of the CCI-MRS2179 group may represent the P2Y1 receptor blockade effects on lowering the metabolic activity of cells, although the post-injury changes suggesting neuronal hyperactivity (decreased glucose with increased lactate) were not different among the CCI groups. A previous in vitro study showed that ATP stimulation of the P2 receptor on astrocytes activates the phosphoinositide 3 kinase/Akt pathway, which in turn depletes intracellular glucose, thus leading to glucose import into the cell via the glucose transporter (56). Blockade of the P2 receptor with MRS2179 may represent the inactivation of this pathway, which resulted in the elevated extracellular glucose level observed in the current study. In contrast to treatment with MRS2179, 2-APB treatment led to a decrease in pre-injury extracellular glucose levels. However, store-operated calcium channel blockade did not exacerbate nor attenuate the post-CCI induced reduction in glucose or increase in lactate compared with those of CCI alone. Blocking the store-operated calcium channel in several injury models has been reported to be beneficial. For example, 2-APB increased ischemic tolerance in a neuron astrocyte co-culture model (57), and inhibition of store-operated calcium channels restored mitochondrial membrane potential, reduced cytochrome $c$ release and prevented cell death in a hyperglycemia-induced in vitro neuronal injury model (58). In contrast to the current results, previous studies indicated that store-operated calcium channels function to increase glucose uptake from the extracellular space, and blockade of these channels with 2-APB will increase the extracellular glucose concentration $(57,59)$. Further studies will be required to explain these contradictory findings and to clarify the roles of store-operated calcium channels on cerebral metabolism or energy status following injury.

In conclusion, the present results demonstrated that post-traumatic ATP signaling can be altered to certain degree by pharmacological interventions. Further studies on pharmacological ways of altering the activation states of astrocytes for treating TBI are warranted.

\section{Acknowledgements}

Not applicable.

\section{Funding}

The current study was supported by the UCLA Brain Injury Research Center.

\section{Availability of data and materials}

The datasets used and/or analyzed during the current study are available from the corresponding author on reasonable request.

\section{Authors' contributions}

NM designed the study and RLS obtained funding. NM and SSG performed the experiments and NM, SSG and RLS analyzed the samples. NM and RLS confirm the authenticity 
of all raw data. NM and RLS wrote the manuscript. All authors read, edited and approved the final manuscript.

\section{Ethics approval and consent to participate}

All experimental procedures were approved by the UCLA Chancellor's Committee for Animal Research. This research was conducted in accordance with the National Institutes of Health guide for the care and use of laboratory animals.

\section{Patient consent for publication}

Not applicable.

\section{Competing interests}

The authors declare that they have no competing interests.

\section{References}

1. Bazargani $\mathrm{N}$ and Attwell D: Amines, astrocytes, and arousal. Neuron 94: 228-231, 2017

2. Harada K, Kamiya T and Tsuboi T: Gliotransmitter release from astrocytes: Functional, developmental, and pathological implications in the brain. Front Neurosci 9: 499, 2015.

3. De Pittà M, Brunel N and Volterra A: Astrocytes: Orchestrating synaptic plasticity? Neuroscience 323: 43-61, 2016.

4. Lalo U, Bogdanov A and Pankratov Y: Age- and experiencerelated plasticity of ATP-mediated signaling in the neocortex. Front Cell Neurosci 13: 242, 2019.

5. Schwarz Y, Zhao N, Kirchhoff F and Bruns D: Astrocytes control synaptic strength by two distinct v-SNARE-dependent release pathways. Nat Neurosci 20: 1529-1539, 2017.

6. Alberini CM, Cruz E, Descalzi G, Bessieres B and Gao V: Astrocyte glycogen and lactate: New insights into learning and memory mechanisms. Glia 66: 1244-1262, 2018.

7. Henneberger C, Papouin T, Oliet SH and Rusakov DA: Long-term potentiation depends on release of D-serine from astrocytes. Nature 463: 232-236, 2010.

8. Cai C, Zambach SA, Fordsmann JC, Lonstrup M, Thomsen KJ Jensen AGK and Lauritzen M: In vivo three-dimensional two-photon microscopy to study conducted vascular responses by local ATP ejection using a glass micro-pipette. J Vis Exp 148: 2019.

9. Haydon PG and Carmignoto G: Astrocyte control of synaptic transmission and neurovascular coupling. Physiol Rev 86: 1009-1031, 2006.

10. Takano T, Tian GF, Peng W, Lou N, Libionka W, Han X and Nedergaard M: Astrocyte-mediated control of cerebral blood flow. Nat Neurosci 9: 260-267, 2006.

11. Corps KN, Roth TL and McGavern DB: Inflammation and neuroprotection in traumatic brain injury. JAMA Neurol 72: 355-362, 2015.

12. Davalos D, Grutzendler J, Yang G, Kim JV, Zuo Y, Jung S, Littman DR, Dustin ML and Gan WB: ATP mediates rapid microglial response to local brain injury in vivo. Nat Neurosci 8: 752-758, 2005.

13. Jassam YN, Izzy S, Whalen M, McGavern DB and El Khoury J: Neuroimmunology of traumatic brain injury: Time for a paradigm shift. Neuron 95: 1246-1265, 2017.

14. Karve IP, Taylor JM and Crack PJ: The contribution of astrocytes and microglia to traumatic brain injury. Br J Pharmacol 173: 692-702, 2016

15. Koizumi S, Shigemoto-Mogami Y, Nasu-Tada K, Shinozaki Y, Ohsawa K, Tsuda M, Joshi BV, Jacobson KA, Kohsaka S and Inoue $\mathrm{K}$ : UDP acting at $\mathrm{P} 2 \mathrm{Y} 6$ receptors is a mediator of microglial phagocytosis. Nature 446: 1091-1095, 2007.

16. Haynes SE, Hollopeter G, Yang G, Kurpius D, Dailey ME, Gan WB and Julius D: The P2Y12 receptor regulates microglial activation by extracellular nucleotides. Nat Neurosci 9: 1512-1519, 2006

17. Winkler U, Seim P, Enzbrenner Y, Köhler S, Sicker M and Hirrlinger J: Activity-dependent modulation of intracellular ATP in cultured cortical astrocytes. J Neurosci Res 95: 2172-2181, 2017.
18. Melani A, Turchi D, Vannucchi MG, Cipriani S, Gianfriddo M and Pedata F: ATP extracellular concentrations are increased in the rat striatum during in vivo ischemia. Neurochem Int 47 442-448, 2005.

19. Wang X, Arcuino G, Takano T, Lin J, Peng WG, Wan P, Li P, Xu Q, Liu QS, Goldman SA and Nedergaard M: P2X7 receptor inhibition improves recovery after spinal cord injury. Nat Med 10: 821-827, 2004

20. Hinzman JM, Wilson JA, Mazzeo AT, Bullock MR and Hartings JA: Excitotoxicity and metabolic crisis are associated with spreading depolarizations in severe traumatic brain injury patients. J Neurotrauma 33: 1775-1783, 2016.

21. Moro N, Ghavim SS, Harris NG, Hovda DA and Sutton RL: Pyruvate treatment attenuates cerebral metabolic depression and neuronal loss after experimental traumatic brain injury. Brain Res 1642: 270-277, 2016.

22. Shijo K, Sutton RL, Ghavim SS, Harris NG and Bartnik-Olson BL: Metabolic fate of glucose in rats with traumatic brain injury and pyruvate or glucose treatments: A NMR spectroscopy study. Neurochem Int 102: 66-78, 2017.

23. Taylor AN, Tio DL, Paydar A and Sutton RL: Sex differences in thermal, stress, and inflammatory responses to minocycline administration in rats with traumatic brain injury. J Neurotrauma 35: 630-638, 2018.

24. Kawamura M, Gachet C, Inoue $\mathrm{K}$ and Kato F: Direct excitation of inhibitory interneurons by extracellular ATP mediated by $\mathrm{P} 2 \mathrm{Y} 1$ receptors in the hippocampal slice. J Neurosci 24: 10835-10845, 2004.

25. Bowser DN and Khakh BS: Vesicular ATP is the predominant cause of intercellular calcium waves in astrocytes. J Gen Physiol 129: 485-491, 2007.

26. Heinke B and Sandkuhler J: Group I metabotropic glutamate receptor-induced $\mathrm{Ca}(2+)$-gradients in rat superficial spinal dorsal horn neurons. Neuropharmacology 52: 1015-1023, 2007.

27. Singaravelu K, Lohr C and Deitmer JW: Regulation of storeoperated calcium entry by calcium-independent phospholipase A2 in rat cerebellar astrocytes. J Neurosci 26: 9579-9592, 2006.

28. Fields RD: Nonsynaptic and nonvesicular ATP release from neurons and relevance to neuron-glia signaling. Semin Cell Dev Biol 22: 214-219, 2011.

29. Illes P, Burnstock G and Tang Y: Astroglia-derived ATP modulates CNS neuronal circuits. Trends Neurosci 42: 885-898, 2019.

30. Loiola EC and Ventura AL: Release of ATP from avian Müller glia cells in culture. Neurochem Int 58: 414-422, 2011.

31. Muller MS and Taylor CW: ATP evokes $\mathrm{Ca}^{2+}$ signals in cultured foetal human cortical astrocytes entirely through $\mathrm{G}$ protein-coupled P2Y receptors. J Neurochem 142: 876-885, 2017.

32. Molnar T, Dobolyi A, Nyitrai G, Barabas P, Heja L, Emri Z, Palkovits $M$ and Kardos J: Calcium signals in the nucleus accumbens: Activation of astrocytes by ATP and succinate. BMC Neurosci 12: 96, 2011.

33. Svobodova I, Bhattaracharya A, Ivetic $M$, Bendova $Z$ and Zemkova H: Circadian ATP release in organotypic cultures of the rat suprachiasmatic nucleus is dependent on P2X7 and P2Y receptors. Front Pharmacol 9: 192, 2018.

34. Guo H, Liu ZQ, Zhou H, Wang ZL, Tao YH and Tong Y: P2Y1 receptor antagonists mitigate oxygen and glucose deprivationinduced astrocyte injury. Mol Med Rep 17: 1819-1824, 2018.

35. Koss DJ, Riedel G and Platt B: Intracellular $\mathrm{Ca}^{2+}$ stores modulate SOCCs and NMDA receptors via tyrosine kinases in rat hippocampal neurons. Cell Calcium 46: 39-48, 2009.

36. Papanikolaou M, Lewis A and Butt AM: Store-operated calcium entry is essential for glial calcium signalling in CNS white matter. Brain Struct Funct 222: 2993-3005, 2017.

37. Koizumi S, Fujishita K, Tsuda M, Shigemoto-Mogami Y and Inoue $\mathrm{K}$ : Dynamic inhibition of excitatory synaptic transmission by astrocyte-derived ATP in hippocampal cultures. Proc Natl Acad Sci USA 100: 11023-11028, 2003.

38. Diniz C, Rodrigues M, Casarotto PC, Pereira VS, Crestani CC and Joca SRL: Monoamine involvement in the antidepressant-like effect induced by P2 blockade. Brain Res 1676: 19-27, 2017.

39. Asadollahi $M$ and Simani L: The diagnostic value of serum UCHL-1 and S100-B levels in differentiate epileptic seizures from psychogenic attacks. Brain Res 1704: 11-15, 2019.

40. Lerchundi R, Huang N and Rose CR: Quantitative imaging of changes in astrocytic and neuronal adenosine triphosphate using two different variants of ATeam. Front Cell Neurosci 14: 80, 2020 . 
41. Burda JE, Bernstein AM and Sofroniew MV: Astrocyte roles in traumatic brain injury. Exp Neurol 275: 305-315, 2016.

42. Wellmann M, Alvarez-Ferradas C, Maturana CJ, Saez JC and Bonansco C: Astroglial $\mathrm{Ca}^{2+}$-dependent hyperexcitability requires $\mathrm{P}_{2} \mathrm{Y}_{1}$ purinergic receptors and pannexin-1 channel activation in a chronic model of epilepsy. Front Cell Neurosci 12. 446, 2018.

43. Sakuragi S, Niwa F, Oda Y, Mikoshiba $\mathrm{K}$ and Bannai $\mathrm{H}$ : Astroglial $\mathrm{Ca}^{2+}$ signaling is generated by the coordination of $\mathrm{IP}_{3} \mathrm{R}$ and store-operated $\mathrm{Ca}^{2+}$ channels. Biochem Biophys Res Commun 486: 879-885, 2017.

44. Li JH, Zhao ST, Wu CY, Cao X, Peng MR, Li SJ, Liu XA and Gao TM: Store-operated $\mathrm{Ca}^{2+}$ channels blockers inhibit lipopolysaccharide induced astrocyte activation. Neurochem Res 38: 2216-2226, 2013

45. Charles AC, Dirksen ER, Merrill JE and Sanderson MJ: Mechanisms of intercellular calcium signaling in glial cells studied with dantrolene and thapsigargin. Glia 7: 134-145, 1993.

46. Obrenovitch TP and Urenjak J: Is high extracellular glutamate the key to excitotoxicity in traumatic brain injury? J Neurotrauma 14: 677-698, 1997.

47. Jourdain P, Bergersen LH, Bhaukaurally K, Bezzi P, Santello M, Domercq M, Matute C, Tonello F, Gundersen V and Volterra A: Glutamate exocytosis from astrocytes controls synaptic strength. Nat Neurosci 10: 331-339, 2007.

48. Parri R and Crunelli V: Astrocytes target presynaptic NMDA receptors to give synapses a boost. Nat Neurosci 10: 271-273, 2007.

49. Rose CR, Felix L, Zeug A, Dietrich D, Reiner A and Henneberger C: Astroglial glutamate signaling and uptake in the hippocampus. Front Mol Neurosci 10: 451, 2017.

50. Dorsett CR, McGuire JL, DePasquale EA, Gardner AE, Floyd CL and McCullumsmith RE: Glutamate neurotransmission in rodent models of traumatic brain injury. J Neurotrauma 34: 263-272, 2017.

51. Gonzalez-Sanchez P, Del Arco A, Esteban JA and Satrustegui J: Store-operated calcium entry is required for mGluR-dependent long term depression in cortical neurons. Front Cell Neurosci 11: 363,2017

52. Carpenter KL, Jalloh I and Hutchinson PJ: Glycolysis and the significance of lactate in traumatic brain injury. Front Neurosci 8: $112,2015$.
53. Zeiler FA, Thelin EP, Helmy A, Czosnyka M, Hutchinson PJA and Menon DK: A systematic review of cerebral microdialysis and outcomes in TBI: Relationships to patient functional outcome, neurophysiologic measures, and tissue outcome. Acta Neurochir (Wien) 159: 2245-2273, 2017.

54. Jones DA, Ros J, Landolt H, Fillenz M and Boutelle MG: Dynamic changes in glucose and lactate in the cortex of the freely moving rat monitored using microdialysis. J Neurochem 75: 1703-1708, 2000.

55. Clausen F, Hillered L and Gustafsson J: Cerebral glucose metabolism after traumatic brain injury in the rat studied by 13C-glucose and microdialysis. Acta Neurochir (Wien) 153: 653-658, 2011

56. Prebil M, Chowdhury HH, Zorec R and Kreft M: Changes in cytosolic glucose level in ATP stimulated live astrocytes. Biochem Biophys Res Commun 405: 308-313, 2011.

57. Tanaka M, Kawahara K, Kosugi T, Yamada T and Mioka T: Changes in the spontaneous calcium oscillations for the development of the preconditioning-induced ischemic tolerance in neuron/astrocyte co-culture. Neurochem Res 32: 988-1001, 2007.

58. Xu Z, Xu W, Song Y, Zhang B, Li F and Liu Y: Blockade of store-operated calcium entry alleviates high glucose-induced neurotoxicity via inhibiting apoptosis in rat neurons. Chem Biol Interact 254: 63-72, 2016.

59. Olianas MC, Dedoni S and Onali P: Involvement of storeoperated $\mathrm{Ca}(2+)$ entry in activation of AMP-activated protein kinase and stimulation of glucose uptake by M3 muscarinic acetylcholine receptors in human neuroblastoma cells. Biochim Biophys Acta 1843: 3004-3017, 2014.

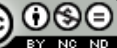

This work is licensed under a Creative Commons
Attribution-NonCommercial-NoDerivatives 4.0 Attribution-NonCommercial-NoDeriv
International (CC BY-NC-ND 4.0) License. 